\title{
Enterococcus durans
}

National Cancer Institute

\section{Source}

National Cancer Institute. Enterococcus durans. NCI Thesaurus. Code C86367.

A species of facultatively anaerobic, Gram positive, cocci shaped bacteria in the phylum Firmicutes. This species is non-motile, ferments melibiose, does not ferment arabinose or citrate, does not reduce tetrazolium and is a lactic acid bacteria. E. durans is pathogenic in chickens but is rarely shown to be pathogenic in humans. 